\title{
Catalytic Enantio- and Diastereoselective Mannich Addition of TosMIC to Ketimines
}

\author{
Allegra Franchino, ${ }^{[a]}$ Jack Chapman, ${ }^{[a]}$ Ignacio Funes-Ardoiz, ${ }^{[b]}$ Robert S. Paton ${ }^{\star[a]}$ and Darren J. \\ Dixon*[a]
}

\begin{abstract}
Chiral amines bearing a stereocenter in the a position are ubiquitous compounds with many applications in the pharmaceutical and agrochemical sectors, as well as in catalysis. Catalytic asymmetric Mannich additions represent a valuable method to access such compounds in enantioenriched form. Herein, we report the first enantio- and diastereoselective addition of commercially available $p$-toluenesulfonylmethyl isocyanide (TosMIC) to ketimines, affording 2-imidazolines bearing two contiguous stereocenters, one of which is fully-substituted, with high yields and excellent stereocontrol. The reaction, catalyzed by silver oxide and a dihydroquinine-derived $N, P$-ligand, is broad in scope, operationally simple and scalable. Derivatization of the products provides enantioenriched vicinal diamines, precursors to $\mathrm{NHC}$ ligands and $\mathrm{sp}^{3}$-rich heterocyclic scaffolds. Computations are used to understand catalysis and rationalize stereoselectivity.
\end{abstract}

Amines bearing a fully-substituted stereocentre in the a position find many applications in the pharmaceutical and agrochemical industry ${ }^{[1]}$ as well as in catalysis. ${ }^{[2]}$ Commensurate with their importance and abundance, numerous methods have been developed for their preparation, ${ }^{[1,3]}$ among which a prominent approach exploits the enantioselective addition of carbanion equivalents to imines derived from prochiral ketones. ${ }^{[4]}$ Ketimines are much more challenging electrophiles than their aldimine counterparts, due to their lower electrophilicity and the more difficult enantiodiscrimination between the two prochiral faces. ${ }^{[4 d-e]}$ Nevertheless, in recent years impressive progress in the development of catalytic enantioselective additions of various pronucleophiles to ketimines has been made. ${ }^{[4,5]}$ Within this context, our group has developed a catalytic system comprising a basic silver(I) salt and a cinchona-derived amino phosphine ligand, able to promote the highly diastereo- and enantioselective addition of isocyanoacetate esters to aldehydes ${ }^{[6]}$ ketones $^{[7]}$ and ketimines (Scheme 1a).$^{[8]}$

Wishing to extend the structural diversity of compounds accessible with this chemistry, we became interested in expanding the scope of the pronucleophile component. Among activated isocyanides, ${ }^{[9]}$ i.e. possessing an electron-withdrawing group in the a position, $p$-toluenesulfonylmethyl isocyanide (TosMIC) presents a series of advantages that were particularly attractive. Unlike most isocyanides, TosMIC is a commercially

[a] Dr. A. Franchino, J. Chapman, Prof. Dr. R. S. Paton, Prof. Dr. D. J. Dixon - Department of Chemistry, Chemistry Research Laboratory University of Oxford, 12 Mansfield Road, Oxford OX1 3TA (UK) E-mail: darren.dixon@chem.ox.ac.uk; robert.paton@chem.ox.ac.uk Dr I. Funes-Ardoiz - Institute of Chemical Research of Catalonia (ICIQ), Av. Països Catalans 16, Tarragona, Spain

Supporting information for this article is given via a link at the end of the document. available, practically odorless, stable, crystalline solid. Its use in organic synthesis has been pioneered in the 1970s by Van Leusen and co-workers. ${ }^{[10]}$ Despite the multifaceted chemistry of TosMIC, only three catalytic enantioselective aldol reactions of this isocyanide have been reported. ${ }^{[11]}$ In 2015 Cozzi and coworkers described the first, and to date only, catalytic enantioselective addition of TosMIC to ketones (Scheme 1b). ${ }^{[11 c]}$ Surprisingly, no enantioselective Mannich reaction of TosMIC has yet been reported, and therefore the potential utility of this practical, versatile $\mathrm{N}$-containing building block for the synthesis of chiral amines has remained untapped. To this end, we aimed to develop such an enantioselective Mannich reaction using ketimine pronucleophiles. We hoped that our $\mathrm{Ag}(\mathrm{I}) / \mathrm{amino}$ phosphine combination (Scheme 1c) would provide both the necessary reactivity and stereocontrol and herein we wish to report our findings.

(a) Previous work: isocyanoacetates and ketimines (Dixon 2014) ${ }^{8 a}$

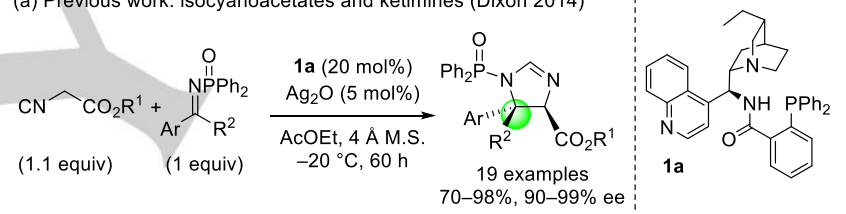

(b) Previous work: TosMIC and ketones (Cozzi 2015) ${ }^{11 \mathrm{C}}$

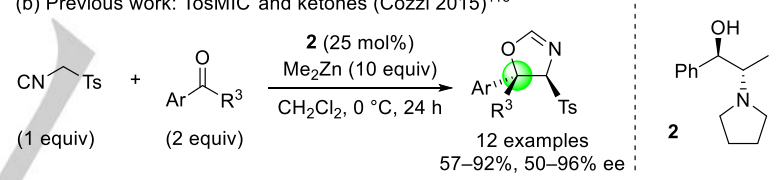

(c) This work: TosMIC and ketimines

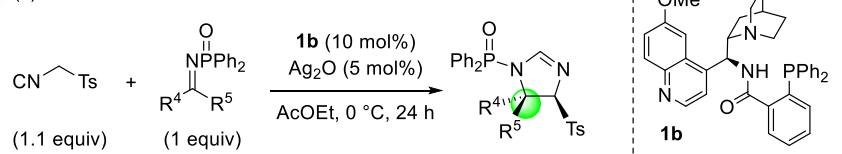

Scheme 1. Catalytic enantio- and diastereoselective aldol and Mannich reactions of activated isocyanides affording products with a fully-substituted stereocenter. (a, b): Previous work. (c): This work.

A model reaction between $N$-diphenylphosphinoyl ( $N$-DPP) acetophenone-derived ketimine $\mathbf{3 a}$ and TosMIC was initially investigated in ethyl acetate as solvent, using $5 \mathrm{~mol} \%$ silver oxide and 10 mol\% dihydroquinine-derived ligand $\mathbf{1 b}$ (Table 1). After 24 hours at room temperature the desired trans imidazoline 5a was obtained as a single diastereomer with very good yield and enantiocontrol (89\% yield, $84 \%$ ee, Entry 1$)$. Lowering the temperature to $0{ }^{\circ} \mathrm{C}$ improved both yield and enantioselectivity (98\% yield, $94 \%$ ee, Entry 2). At this temperature, decreasing the $\mathrm{Ag}_{2} \mathrm{O}$ loading had a slight detrimental effect on enantiocontrol (Entry 3), whereas dilution of the reaction mixture increased the ee of the product to $96 \%$ (Entry 4). The use of 10 mol\% silver acetate in conjunction with $10 \mathrm{~mol} \% \mathbf{1 b}$ promoted the reaction with comparable enantioselectivity but diminished yield, owing to a competitive cyclodimerization of TosMIC to imidazole 6. ${ }^{[12]}$ 
Table 1. Optimization of the silver-catalyzed enantioselective Mannich reaction of ketimine $\mathbf{3} \mathbf{a}$ and TosMIC

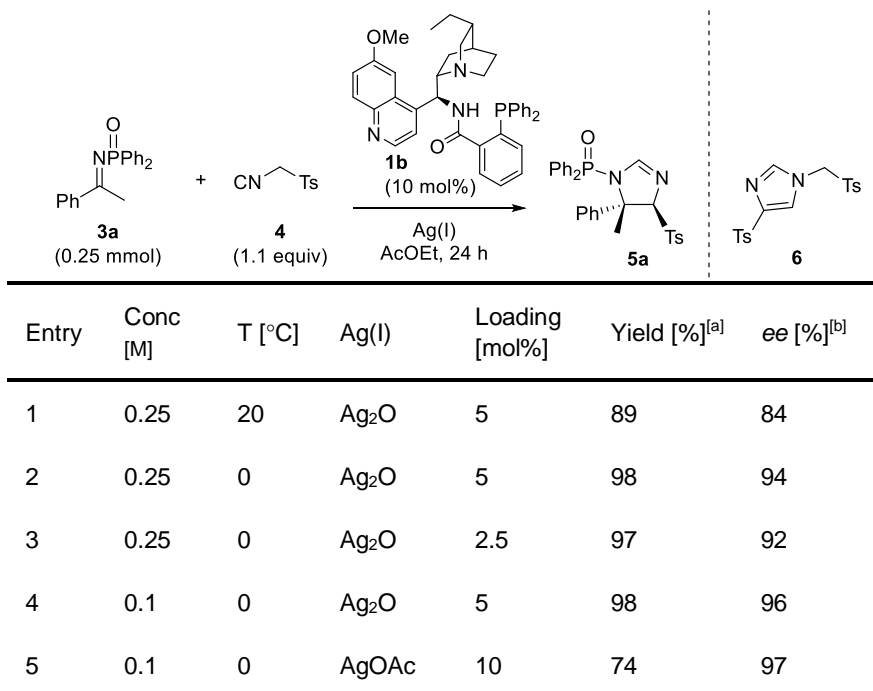

[a] Isolated yield for the trans diastereomer after FCC. [b] ee for the trans diastereomer determined by HPLC on chiral stationary phase.

With optimal conditions established, substituents on the $N$-DPP ketimine were systematically varied to assess the substrate scope of the methodology (Scheme 2). All reactions proceeded to full conversion within 24 hours yielding the trans imidazoline as the only or largely major product ( $\mathrm{dr}$ of the crude reaction mixture $\geq 90: 10$ ). Aryl methyl ketimines with electron-donating groups (OMe), as well as electron-withdrawing substituents ( $F$ $\mathrm{Cl}, \mathrm{Br}$ ) in the para, meta and ortho positions afforded products 5b-h with high yields and excellent enantiocontrol (86-97\% yield, $86-97 \%$ ee). While the small fluoro substituent in ortho position was well-tolerated, a double loading of both ligand and $\mathrm{Ag}_{2} \mathrm{O}$ was required to ensure full conversion for the reaction of ketimine $\mathbf{3 d}$ bearing an o-methoxyphenyl group. Heteroaromatic ketimines reacted smoothly and importantly imidazolines with 4pyridyl, 3-pyridyl and 2-pyridyl substituents were prepared in 86$99 \%$ yield and $90-95 \%$ ee (5i-k). Products $5 \mathrm{I}$ and $\mathbf{5 m}$ with 2 furanyl and 2-thienyl substitutents were obtained in $82 \%$ yield and $98 \%$ ee, $98 \%$ yield and $96 \%$ ee, respectively. In addition, it was possible to increase the length of the alkyl chain on the ketimine delivering product $5 \mathrm{n}$ with a $n$-propyl group in $88 \%$ yield and $93 \%$ ee; interestingly, a phenyl isobutyl ketimine with a $\beta$ branched alkyl chain was completely unreactive. Aliphatic ketimines with primary, secondary and even tertiary alkyl groups were competent electrophiles. Aliphatic ketimines with phenethyl and cyclohexyl substituents afforded products 50 and $5 p$ in very high yield and ee. Forcing conditions were necessary to accomplish the addition of TosMIC to tert-butyl methyl ketimine, obtaining $\mathbf{5 q}$ in $51 \%$ yield and $83 \%$ ee, still a remarkable result considering the steric and electronic deactivation of this substrate. ${ }^{[13]}$

Single crystal X-ray diffraction allowed the determination of the absolute configuration for the major enantiomer of imidazoline 5e $\left(4 S, 5 S\right.$, Scheme 2); ${ }^{[14]}$ the relative and absolute configurations of the other reaction products were assigned by analogy. NOe experiments carried out on products $\mathbf{5 0}$ and $\mathbf{5 q}$, and separately on both diastereomers of imidazolines $\mathbf{5 k}$ and $\mathbf{5 I}$ confirmed the relative trans stereochemistry also for imidazolines with aliphatic and heteroaromatic substituents.

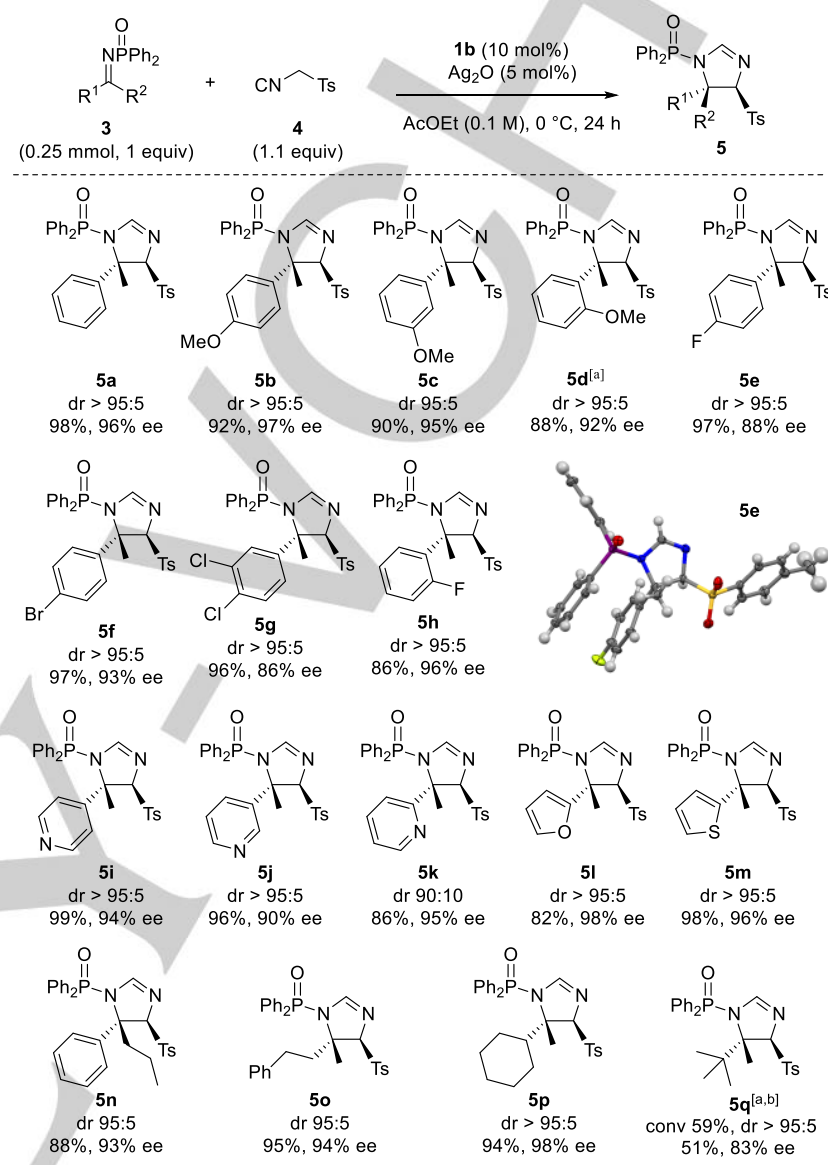

Scheme 2. Substrate scope for the catalytic diastereo- and enantioselective addition of TosMIC to N-DPP ketimines. Dr (trans:cis) of the crude reaction mixture determined by ${ }^{1} \mathrm{H}$ NMR analysis. Isolated yields and ee given for the trans diastereomer after FCC. ORTEP diagram for 5 e with ellipsoids at $50 \%$ probability level. [a] With $20 \mathrm{~mol} \% \mathbf{1 b}$ and $10 \mathrm{~mol} \% \mathrm{Ag}_{2} \mathrm{O}$. [b] At $0.25 \mathrm{M}$ concentration for 120 .

As well as being operationally simple, the catalytic enantioselective reaction between ketimine $\mathbf{3 a}$ and TosMIC also proved to be scalable. While the substrate scope was conducted on a $0.25 \mathrm{mmol}$ scale, reoptimization for preparative scale $(10$ $\mathrm{mmol}$ ) allowed for a reduction in the catalyst loading to $2 \mathrm{~mol} \%$ and a relaxation of the strictly anhydrous conditions. Thus, nearly stoichiometric amounts of the two reagents were mixed with $2 \mathrm{~mol} \% \mathbf{~} \mathbf{b}$ and $1 \mathrm{~mol} \%$ silver oxide in ethyl acetate and stirred at room temperature under air in an open vessel for 24 hours, to obtain a full conversion to the trans imidazoline. After dilution and filtration of the crude reaction mixture to remove insoluble silver residues, the filtrate was conveniently purified by recrystallization. This conveniently resulted in a concomitant augmentation of the enantiomeric excess, thus delivering the desired product $\mathbf{5 a}$ in $78 \%$ yield and greater than $99.5 \%$ ee (Scheme 3). 


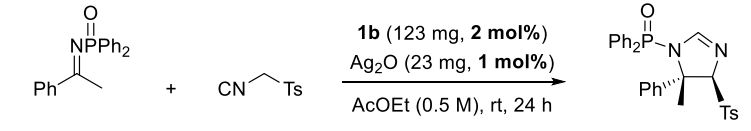

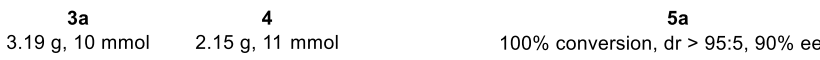

$$
\begin{aligned}
& \text { recrystallization }
\end{aligned}
$$

AcOEt/hexane: $4.56 \mathrm{~g}, \mathbf{8 9} \%$ yield, $\mathbf{9 9} \%$ ee or $\mathrm{CH}_{2} \mathrm{Cl}_{2} / \mathrm{AcOEt}: 3.88 \mathrm{~g}, \mathbf{7 8 \%}$ yield, $>\mathbf{9 9 . 5 \%}$ ee

Scheme 3. Multigram synthesis of imidazoline 5a (40-fold scale-up).

With gram quantities of enantiopure imidazoline $5 \mathrm{a}$ in hand, we focused on its derivatization into useful products to thus demonstrate the utility of our catalytic enantioselective methodology (Scheme 4). To this end, treatment of 5a with excess lithium aluminium hydride removed both the DPP and the tosyl groups affording 7 in $80 \%$ yield without affecting the enantiopurity. The deprotected imidazoline could be hydrolysed under basic conditions, obtaining diamine $\mathbf{8}$, or methylated on both $\mathrm{N}$ atoms to give imidazolium iodide $\mathbf{9}$, a precursor to a nonsymmetrical $\mathrm{N}$-heterocyclic carbene (NHC). Several acidic conditions were tried to cleave the DPP group ${ }^{[15]}$ but the tosyl group was not stable to the reaction conditions. When the $\mathrm{N}$ protected imidazoline 5 a was treated with aqueous $\mathrm{HCl}$, 4hydroxyl imidazolium chloride $\mathbf{1 0}$ was obtained as a 3:2 mixture of epimers at C-4 in $40 \%$ yield (single crystal X-ray diffraction of the trans diastereomer allowed structural identification). ${ }^{[14,16]}$ Treatment of $\mathbf{1 0}$ with sodium hydroxide provided $C_{2}$-symmetric dihydropyrazine 12 via dimerization of $\alpha$-amino aldehyde 11. ${ }^{[17]}$ A two-step, one-pot procedure was developed to avoid isolation of 10 , obtaining dihydropyrazine 12 in $86 \%$ yield from 5 a without ee erosion.

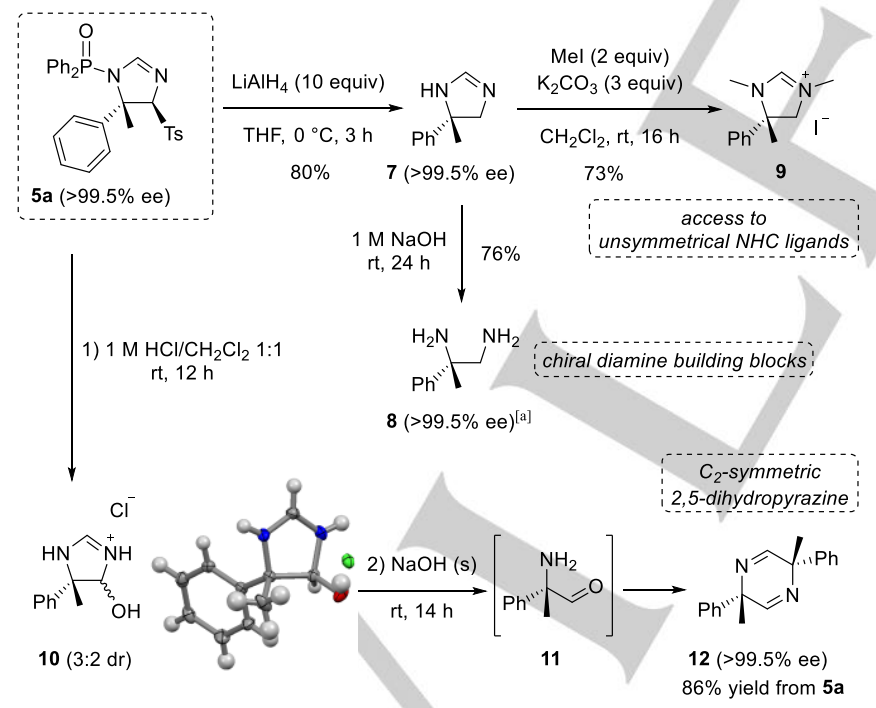

Scheme 4. Synthetic utility of imidazoline 5a. ORTEP diagram for 10 (trans diastereomer) shown with ellipsoids at $50 \%$ probability level. [a] Ee of diamine 8 determined on the corresponding ditosylamide.

In order to better understand the importance of the various functional groups present on amino phosphine $\mathbf{1 b}$, ligands with structural variations were screened in the model reaction (Table 2). While quinine-derived amino phosphine $\mathbf{1 b}$ gave product $\mathbf{5 a}$ in $98 \%$ yield and $96 \%$ ee (entry 1), cinchonidine-derived 1a, lacking the methoxy group, and quinine-derived 1c with a non- hydrogenated side chain showed slightly lower reactivity and enantioselectivity (entries 2 and 3). Ligand 1d devoid of the phosphine moiety delivered the product in only $8 \%$ yield and in racemic form (entry 4 ), and the addition of $10 \mathrm{~mol} \%$ exogenous triphenylphosphine did not improve its performance significantly (entry 5), highlighting the importance of a bidentate $N, P$-ligand for reactivity and stereocontrol. Amino phosphine 1e with a methylated amide linker promoted the reaction with diminished reactivity, and lower diastereo- and enantioselectivity (61\% conversion, $83: 17 \mathrm{dr}, 88 \%$ ee, entry 6 ), suggesting that the $\mathrm{NH}$ of the linker plays a role in the reaction. ${ }^{[\mathrm{bb}]}$ Ligand $1 \mathrm{f}$ possessing an ester linker afforded product ent-5a in 50\% yield with $90 \%$ ee (entry 7): replacement of the $\mathrm{NH}$ group by an $\mathrm{O}$ atom decreased the reactivity and imparted a switch in the enantiofacial selectivity for both reaction partners, even if ligands $\mathbf{1 b}$ and $\mathbf{1 f}$ on their own display nearly superimposable crystal structures. ${ }^{[14]}$
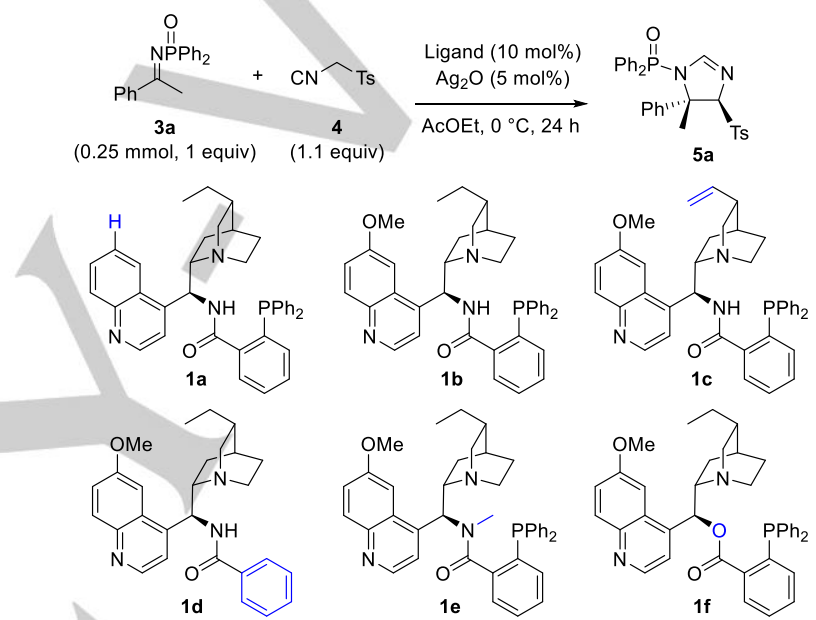

Table 2. Ligand variation

\begin{tabular}{|c|c|c|c|c|c|}
\hline Entry & Ligand & Conversion (\%) $)^{[\mathrm{a}]}$ & $\operatorname{Dr}(\%)^{[\mathrm{a}]}$ & Yield $(\%)^{[b]}$ & $e e(\%)^{[b]}$ \\
\hline 1 & $1 a$ & 96 & $>95: 5$ & 88 & 92 \\
\hline 2 & $1 b$ & 100 & $>95: 5$ & 98 & 96 \\
\hline 3 & $1 c$ & 81 & $>95: 5$ & 70 & 93 \\
\hline 4 & 1d & 24 & $33: 67$ & 8 & 0 \\
\hline $5^{[c]}$ & $1 d$ & 53 & $68: 32$ & 34 & -6 \\
\hline 6 & $1 e$ & 61 & $83: 17$ & 51 & 88 \\
\hline 7 & $1 f$ & 54 & $91: 9$ & 50 & -90 \\
\hline
\end{tabular}

[a] Conversion and dr (trans:cis) were determined by ${ }^{1} \mathrm{H}$ NMR analysis of the crude reaction mixture, using mesitylene as an internal standard. [b] Isolated yield and ee of the trans diastereomer. [c] With $10 \mathrm{~mol} \% \mathrm{PPh}_{3}$.

Furthermore, when isolated cis and trans diastereomers of imidazoline 5l were subjected to the standard reaction conditions, no epimerization occurred (see SI for details), indicating that the reaction is not reversible once product formation has occurred, suggesting that the ligand is able to impart significant stereoselectivity in the enantiodetermining $\mathrm{C}-\mathrm{C}$ bond forming step. To rationalize these observations, quantum chemical calculations (DFT and QM/MM) were performed. ${ }^{[18]}$ 
Based on single crystal X-ray diffraction and NMR studies providing evidence of complex formation between $\mathrm{AgOAc}$ and $N, P$-ligand $\mathbf{1 b},{ }^{[8 b]}$ we performed a computational analysis of competing transition structures (TSs) (Figure 2). Since N-DPP ketimines exist as a mixture of $E$ - and Z-configurated stereoisomers rapidly interconverting at room temperature, ${ }^{[19]}$ we examined both configurations computationally. In the most stable TSs the imine adopts an E-configuration, which is favored sterically. In addition to N,P-coordination of silver, the ligand plays a role in substrate activation during $\mathrm{C}-\mathrm{C}$ bond formation: the phosphinoyl oxygen was found to coordinate to the amide $\mathrm{N}$ $\mathrm{H}$ proton in addition to $\mathrm{Ag}$ in each of the most stable TSs (Fig. 2). The major TS is perfectly staggered about the forming C-C bond, whereas steric interactions between the quinuclidine region of the ligand and the ketimine phenyl group result in a less-stable, eclipsed, TS forming the minor enantiomer $\left(\Delta \Delta \mathrm{G}^{\ddagger} 2.3 \mathrm{kcal} / \mathrm{mol}\right)$.

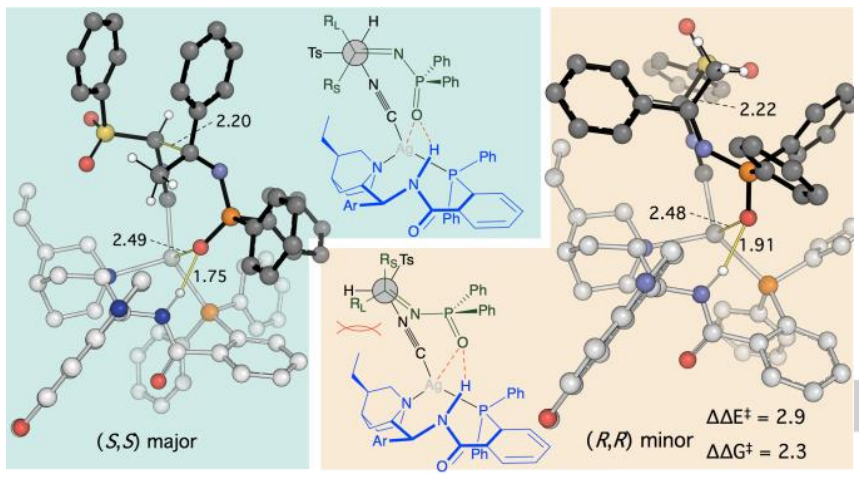

Figure 2. Competing C-C forming TSs for major and minor enantiomers for the reaction between acetophenone-derived $N$-DPP ketimine and TosMIC (SMD-M06/6-311++G(d,p)//ONIOM(M06/def2-SVP:UFF).

In summary, we have developed the first enantioselective addition of the versatile, commercially available $p$ toluenesulfonylmethyl isocyanide to ketimines, by using a $\mathrm{Ag}(\mathrm{I}) /$ amino phosphine catalytic system. The reaction afforded 2 imidazolines with high yields and excellent diastereo- and enantiocontrol, which could be elaborated to vicinal diamines, precursors to NHC ligands and heterocyclic scaffolds such as 2,5-dihydropyrazines. The transformation was broad in scope, encompassing several (hetero)aromatic and aliphatic ketimines, operationally simple and scalable. Computations reveal a bifunctional catalyst with $\mathrm{Ag}$ and $\mathrm{N}-\mathrm{H}$ groups activating the electrophile, and rationalize the experimental stereoselectivity.

\section{Acknowledgements}

The authors thank the European Union for funding ( $\mathrm{PhD}$ fellowship to A.F., FP7/2007-2013, 316955) and Severo Ochoa (predoctoral training fellowship to I. F.-A., SVP-2014- 0686662). We thank Dr Adam D. Gammack Yamagata for useful discussion, Heyao Shi and Dr. Angel Fuentes de Arriba for X-ray analysis and the Oxford Chemical Crystallography Service for use of their instrumentation. This work used the RMACC Summit cluster (NSF ACl-1532235 and $\mathrm{ACl}-1532236)$, supported by the University of Colorado Boulder and Colorado State University.
Keywords: Mannich reaction $\bullet$ isocyanides $•$ addition to ketimines $\bullet$ silver $\bullet$ homogeneous catalysis

[1] Chiral Amine Synthesis: Methods, Developments and Applications (Ed. T. C. Nugent), Wiley, Weinheim, 2010.

[2] S. France, D. J. Guerin, S. J. Miller, T. Lectka, Chem. Rev. 2003, 103, 2985-3012.

[3] A. K. Mailyan, J. A. Eickhoff, A. S. Minakova, Z. Gu, P. Lu, A. Zakarian, Chem. Rev. 2016, 116, 4441-4557.

[4] For reviews on catalytic enantioselective additions to $\mathrm{C}=\mathrm{N}$ bonds, see (a) A. Cordova, Acc. Chem. Res. 2004, 37, 102-112; (b) G. K. Friestad, A. K. Mathies, Tetrahedron 2007, 63, 2541-2569; (c) D. Ferraris, Tetrahedron 2007, 63, 9581-9597; (d) O. Riant, J. Hannedouche, Org. Biomol. Chem. 2007, 5, 873-888; (e) M. Shibasaki, M. Kanai, Chem. Rev. 2008, 108, 2853-2873; (f) K. Yamada, K. Tomioka, Chem. Rev. 2008, 108, 2874-2886; (g) S. Kobayashi, Y. Mori, J. S. Fossey, M. W. Salter, Chem. Rev. 2011, 111, 2626-2704; (h) M. Yus, J. C. GonzálezGómez, F. Foubelo, Chem. Rev. 2011, 111, 7774-7854; (i) Science of Synthesis: Stereoselective Synthesis 2: Stereoselective Reactions of Carbonyl and Imino Groups, (Vol. Ed.: G. A. Molander), Thieme, Stuttgart, 2011; (j) T. Akiyama, in Comprehensive Organic Synthesis, (Ed.: P. Knochel and G. A. Molander), Elsevier, Oxford, $2^{\text {nd }}$ edn, 2014 vol. 2, p. 629; (k) N. Kumagai, M. Shibasaki, Bull. Chem. Soc. Jpn. 2015, 88, 503-517.

[5] For selected examples of catalytic enantioselective additions to ketimines, see: (a) P. Vachal, E. N. Jacobsen, J. Am. Chem. Soc. 2002, 124, 10012-10014; (b) W. Zhuang, S. Saaby, K. A. Jørgensen, Angew. Chem. Int. Ed. 2004, 43, 4476-4478; Angew. Chem. 2004, 116, 45764578; (c) Y. Suto, M. Kanai, M. Shibasaki, J. Am. Chem. Soc. 2007 129, 500-501; (d) C. Tan, X. Liu, L. Wang, J. Wang, X. Feng, Org. Lett. 2008, 10, 5305-5308; (e) T. Kano, S. Song, Y. Kubota, K. Maruoka, Angew. Chem. Int. Ed. 2012, 51, 1191-1194; Angew. Chem. 2012, 124, 1217-1220; (f) T. Nishimura, A. Noishiki, G. C. Tsui, T. Hayashi, J. Am. Chem. Soc. 2012, 134, 5056-5059; (g) S. Nakamura, S. Takahashi, D. Nakane, H. Masuda, Org. Lett. 2015, 17, 106-109; (h) H. Jang, F. Romiti, S. Torker, A. H. Hoveyda, Nat. Chem. 2017, 9, 1269-1275; (i) P. Ortiz, J. F. Collados, R. P. Jumde, E. Otten, S. R. Harutyunyan, Angew. Chem. Int. Ed. 2017, 56, 3041-3044; Angew. Chem. 2017, 129, 30873090.

[6] F. Sladojevich, A. Trabocchi, A. Guarna and D. J. Dixon, J. Am. Chem. Soc. 2011, 133, 1710-1713.

[7] R. de la Campa, I. Ortín, D. J. Dixon, Angew. Chem. Int. Ed. 2015, 54, 4895; Angew. Chem. 2015, 127, 4977-4980.

[8] a) I. Ortín, D. J. Dixon, Angew. Chem. Int. Ed. 2014, 53, 3462-3465; Angew. Chem. 2014, 126, 3530-3533; b) R. de la Campa, A. D Gammack Yamagata, I. Ortín, A. Franchino, A. L. Thompson, B. Odell, D. J. Dixon, Chem. Commun. 2016, 52, 10632-10635.

[9] M. Giustiniano, A. Basso, V. Mercalli, A. Massarotti, E. Novellino, G. C. Tron, J. Zhu, Chem. Soc. Rev. 2017, 46, 1295-1357.

[10] a) D. Van Leusen, A. M. van Leusen, Org. React. 2001, 57, 417-666; b) T. Kaur, P. Wadhwa, A. Sharma, RSC Adv. 2015, 5, 52769-52787.

[11] a) M. Sawamura, H. Hamashima, Y. Ito, J. Org. Chem. 1990, 55, 59355936; b) Y. Motoyama, H. Kawakami, K. Shimozono, K. Aoki, H. Nishiyama, Organometallics 2002, 21, 3408-3416; c) A. R. Keeri, A. Gualandi, A. Mazzanti, J. Lewinski, P. G. Cozzi, Chem. Eur. J. 2015, 21, 18949-18952.

[12] Cyclodimerization of TosMIC under basic conditions was already observed: O. H. Oldenziel, D. Van Leusen, A. M. van Leusen, J. Org. Chem. 1977, 42, 3114-3118.

[13] TosMIC analogues possessing a methyl, ethyl or phenyl group in the a position were not competent pronucleophiles due to a competitive and dominant cyclodimerization reaction (see $\mathrm{SI}$ ). Ketimines possessing an aldehyde or ketone functional group on the aromatic ring are expected to undergo competitive oxazoline formation (see references 6, 7 and 11). A nitro group on the aromatic ring of the ketimine may also 
interfere with the reaction outcome lowering yield and enantioselectivity, as observed in a related isocyanoacetate aldo reaction catalyzed by a $\mathrm{Ag}_{2} \mathrm{O} /$ cinchonine-derived amino phosphine system: A. Franchino, P. Jakubec, D. J. Dixon, Org. Biomol. Chem. 2016, 14, 93-96.

[14] $\operatorname{CCDC} 1840592$ (5e), 1840589 (10), 1840590 (1b) and 1840591 (1f) contain the supplementary crystallographic data for this paper. These data can be obtained free of charge from The Cambridge Crystallographic Data Centre.

[15] R. Ramage, D. Hopton, M. J. Parrott, G. W. Kenner, G. A. Moore, J. Chem. Soc., Perkin Trans. 1 1984, 1357-1370.

[16] The low yield reflects difficulties in the isolation, as the aqueous phase $(1 \mathrm{M} \mathrm{HCl})$ was washed several times with $3: 1 \mathrm{CHCl}_{3} / i-\mathrm{PrOH}$ to remove diphenylphosphinic, $p$-toluenesulfinic and $p$-toluenesulfonic acids.

[17] The formation of aldehyde $\mathbf{1 1}$ is supported by the presence of a peak at $\delta 9.45 \mathrm{ppm}$ in the ${ }^{1} \mathrm{H}$ NMR spectrum $\left(\mathrm{CDCl}_{3}\right)$ of incomplete hydrolysis reactions; over time the peak disappears converting to 12 . In an analogous fashion, 4-tosyl and 4-alkoxy oxazolines afford a-hydroxy aldehydes upon acidic treatment: F. J. A. Hundscheid, V. K. Tandon, P. H. F. M. Rouwette, A. M. van Leusen, Tetrahedron 1987, 28, 50735088.

[18] SMD-M06/6-31G(d)/LANL2DZ(f)//ONIOM(M06/def2-SVP:UFF) calculations were performed using Gaussian 09 rev. D.01, M. J. Frisch et al. Gaussian, Inc., Wallingford CT, 2009. Calculations were performed for the isocyanoacetate aldol reaction [ref. 6] and the present TosMIC addition to ketimines; furthermore, transition states consistent with the major enantiomers observed were computed for $\mathrm{N}$-methylated ligand $\mathbf{1 e}$ and ester ligand 1f. Full details are provided in the SI.

[19] S. Masumoto, H. Usuda, M. Suzuki, M. Kanai, M. Shibasaki, J. Am. Chem. Soc. 2003, 125, 5634-5635. 

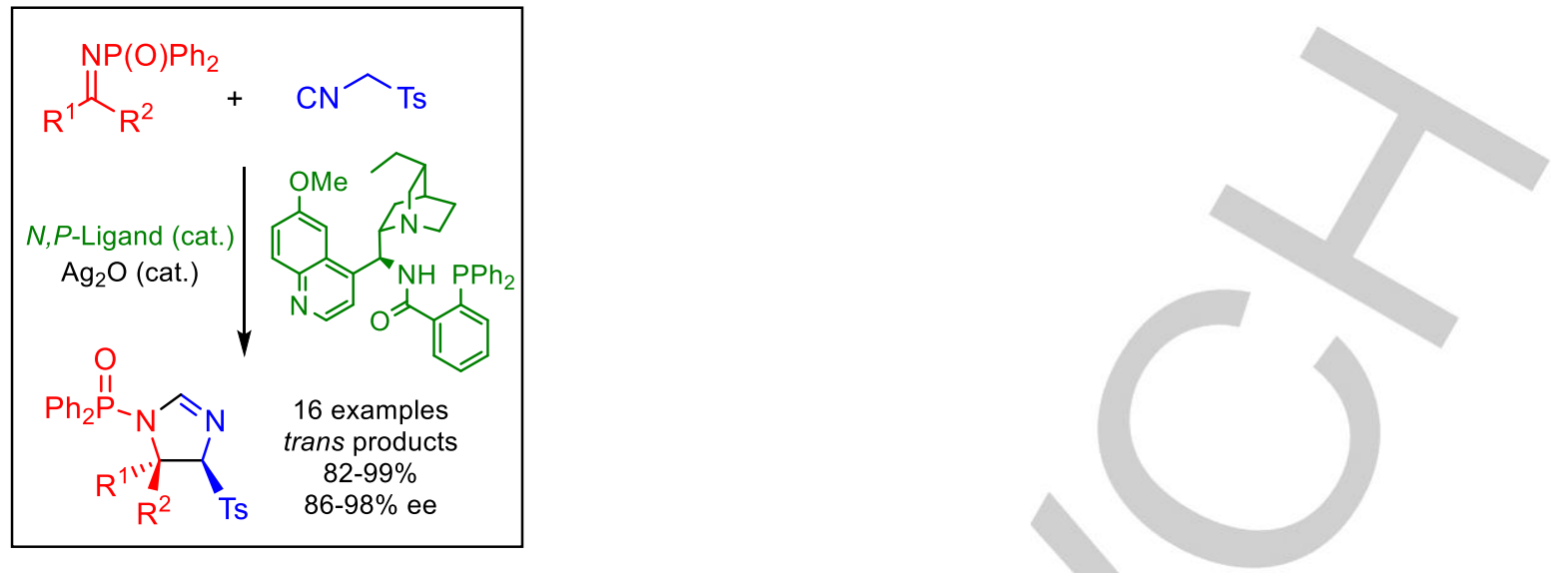

The first enantio- and diastereoselective addition of TosMIC to (hetero)aromatic and aliphatic ketimines provides imidazolines with two contiguous stereocenters, that can be elaborated to vicinal diamines, precursors to NHC ligands and $\mathrm{sp}^{3}$-rich heterocyclic scaffolds. Computations allow to rationalize the stereochemical outcome in reactions catalyzed by silver $(\mathrm{I})$ and a dihydroquinine-derived $N, P$-ligand. 